\title{
Quantum fluctuations of entropy production for fermionic systems in the Landauer-Büttiker state
}

\author{
Mihail Mintchev \\ Istituto Nazionale di Fisica Nucleare and Dipartimento di Fisica dell'Università di Pisa, Largo Pontecorvo 3, 56127 Pisa, Italy \\ Luca Santoni \\ Institute for Theoretical Physics and Center for Extreme Matter and Emergent Phenomena, Utrecht University, \\ Leuvenlaan 4, 3584 CE Utrecht, the Netherlands \\ Paul Sorba \\ LAPTh, Laboratoire d'Annecy-le-Vieux de Physique Théorique, CNRS, Université de Savoie, BP 110, 74941 Annecy-le-Vieux Cedex, France
} (Received 5 June 2017; revised manuscript received 8 October 2017; published 15 November 2017)

\begin{abstract}
The quantum fluctuations of the entropy production for fermionic systems in the Landauer-Büttiker nonequilibrium steady state are investigated. The probability distribution, governing these fluctuations, is explicitly derived by means of quantum field theory methods and analyzed in the zero frequency limit. It turns out that microscopic processes with positive, vanishing and negative entropy production occur in the system with nonvanishing probability. In spite of this fact, we show that all odd moments (in particular, the mean value of the entropy production) of the above distribution are non-negative. This result extends the second principle of thermodynamics to the quantum fluctuations of the entropy production in the Landauer-Büttiker state. The effect of the time reversal is also discussed.
\end{abstract}

DOI: 10.1103/PhysRevE.96.052124

\section{INTRODUCTION}

The entropy production is a measure for irreversibility and represents an essential characteristic feature of nonequilibrium systems. In the quantum context the entropy production is fundamental for understanding the deep interplay between microscopic and macroscopic physics and, in particular, the second principle of thermodynamics. For this reason the study of the entropy production is receiving a constant attention [1-6]. A variety of off-equilibrium states [7-10] and different physical systems [11-16] have been already analyzed. In addition, the fluctuation relations which have been established [17-23] provide universal information about the nature of the entropy production and the related time reversal breaking.

In this article we investigate the entropy production in quantum systems, which are schematically shown in Fig. 1. Each of the two semi-infinite leads $L_{i}$ is attached at infinity to a heat reservoir $R_{i}$ with (inverse) temperature $\beta_{i} \geqslant 0$ and chemical potential $\mu_{i} \in \mathbb{R}$. The capacity of the reservoirs is assumed to be large enough so that the processes of emission and absorption of particles do not change the parameters of $R_{i}$. A pointlike defect is localized at $x=0$ and is described by a unitary scattering matrix $\mathbb{S}$.

The system in Fig. 1 models a quantum wire junction [24-29]. The interest in such devices, which are essentially one-dimensional systems whose transport properties are affected by quantum effects, is largely motivated by the fact that they would naturally appear in any quantum circuit. Triggered by the remarkable progress in nanotechnology, the study of quantum wire junctions nowadays dominates the experimental activity in quantum transport. The focus is mainly on the particle and heat transport, but recently the entropy production in quantum circuits [30] and other mesoscopic systems [31] has attracted much attention as well.

The basic physical processes, taking place in the system in Fig. 1, can be summarized as follows. A nonvanishing transmission probability $\left|\mathbb{S}_{12}\right|^{2}$ drives the system away from equilibrium, provided that the temperatures and/or chemical potentials are different. The departure from equilibrium is characterized by the presence of incoming and outgoing matter and energy flows from the reservoirs $R_{i}$. The study of these flows started with the pioneering work of Landauer [32] and Büttiker [33], who developed an exact scattering approach, going far beyond the linear response approximation. The Landauer-Büttiker (LB) framework is the basis of modern quantum transport theory and has been successfully generalized [34-36] and applied to the computation of the noise power [37-44] and the full counting statistics [45-50].

In what follows we apply the LB approach to the study of the entropy production. We concentrate on fermionic systems, discussing the bosonic case elsewhere [51]. The basic ingredients of our investigation are

(1) a suitably defined field operator $\dot{S}(t, x)$, which describes the entropy production, and

(2) a nonequilibrium steady state $\Omega_{\mathrm{LB}}$, which captures the physical properties of the system shown in Fig. 1.

With this input, all the information about the entropy production is codified in the sequence of $n$-point correlation functions $(n=1,2, \ldots)$,

$$
w_{n}[\dot{S}]\left(t_{1}, x_{1}, \ldots, t_{n}, x_{n}\right)=\left\langle\dot{S}\left(t_{1}, x_{1}\right) \cdots \dot{S}\left(t_{n}, x_{n}\right)\right\rangle_{\mathrm{LB}},
$$

the expectation value $\langle\cdots\rangle_{\mathrm{LB}}$ being computed in the LB state $\Omega_{\mathrm{LB}}$.

Previous research in the quantum context has been mainly focused on $w_{1}[\dot{S}]$, which describes the mean value of the entropy production. Adopting quantum field theory methods, we address in this paper the problem of the quantum fluctuations, which are fully characterized by (1) with $n \geqslant 2$. The correlation functions $w_{n}[\dot{S}]$ depend on $2 n$ space-time variables, which complicate the analysis for large $n$. In order to simplify the problem, we follow the standard approach [43-50] 
to full counting statistics and investigate the zero frequency limit $\mathcal{W}_{n}[\dot{S}]$ of $w_{n}[\dot{S}]$, integrating the quantum fluctuations over a long period of time. We show that in this limit $\mathcal{W}_{n}[\dot{S}]$ take the form

$$
\mathcal{W}_{n}[\dot{S}]=\int_{0}^{\infty} \frac{d \omega}{2 \pi} \mathcal{M}_{n}(\omega),
$$

where $\omega$ is the energy and $\mathcal{M}_{n}$ are the moments of a probability distribution $\varrho$. The derivation of $\varrho$ represents a key point of our investigation. In fact, we extract from $\varrho$ the basic information about the entropy production at the microscopic level. The fundamental quantum process, which takes place in our system, is the emission of a particle from the reservoir $R_{i}$ and the subsequent absorption by $R_{j}$. We derive from $\varrho$ the probability $p_{i j}$ for this event at any energy $\omega$ and determine the corresponding entropy production

$$
\sigma_{i j}=\left[\left(\beta_{i}-\beta_{j}\right) \omega-\left(\beta_{i} \mu_{i}-\beta_{j} \mu_{j}\right)\right]\left|\mathbb{S}_{12}\right| .
$$

In the absence of transmission $\left(\mathbb{S}_{12}=0\right)$ one has $\sigma_{i j}=0$ in agreement with the fact that the two heat reservoirs are disconnected and the system is in equilibrium. The antisymmetry of $\sigma_{i j}$ implies furthermore that the entropy production for emission and absorption of a particle by the same reservoir vanishes, as expected on general grounds. Moreover, $\sigma_{12}$ and $\sigma_{21}$ have opposite sign, which, combined with the fact that $p_{12} \neq 0$ and $p_{21} \neq 0$, leads to the conclusion that both processes with positive and negative entropy production are necessarily present at the microscopic level. Nevertheless, we demonstrate below that the process with positive entropy production dominates in the state $\Omega_{\mathrm{LB}}$, implying that all moments $\left\{\mathcal{M}_{n}(\omega): \omega \geqslant 0, n=1,2, \ldots\right\}$ of $\varrho$ obey

$$
\mathcal{M}_{n}(\omega) \geqslant 0 \text {, }
$$

for any value of the temperatures and chemical potentials of $R_{i}$. In addition, $\mathcal{M}_{n}(\omega)$ vanishes for any $\omega$ only at the equilibrium $\beta_{1}=\beta_{2}$ and $\mu_{1}=\mu_{2}$.

For even $n$ the inequality (4) follows directly from the fact that $\varrho$ is a true probability distribution on $\mathbb{R}$, whereas for odd $n$ it is a consequence of the specific form of $\varrho$. It generalizes to the quantum fluctuations the result of Nenciu [7]

$$
\langle\dot{S}(t, x)\rangle_{\mathrm{LB}}=\int_{0}^{\infty} \frac{d \omega}{2 \pi} \mathcal{M}_{1}(\omega) \geqslant 0
$$

about the mean value of the entropy production in $\Omega_{\mathrm{LB}}$, which provides a bridge between microscopic quantum physics and the second law of thermodynamics. In this respect, the bound (4) represents an extension of the second principle to the quantum fluctuations of the entropy production. The result (4) is an intrinsic characteristic feature of the LB state. To our knowledge no other steady sates with this property are presently known.

The paper is organized as follows. In the next section we describe the basic physical properties of the system. We also introduce the entropy production operator $\dot{S}$ and the LB representation incorporating the nonequilibrium properties of the system in Fig. 1. The $n$-point correlation functions of $\dot{S}$ in the LB state $\Omega_{\mathrm{LB}}$ are derived in Sec. III. In Sec. IV we reconstruct the probability distribution $\varrho$ associated with the entropy production, solving the corresponding moment problem. The physical properties of $\varrho$ are

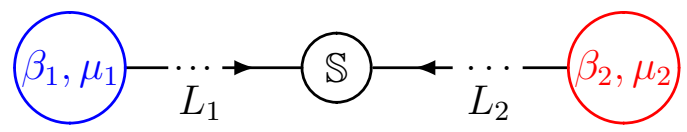

FIG. 1. Two-terminal junction.

discussed, and the role of time reversal is elucidated. It is also shown that the presence of a galvanometer in the system does not modify the bound (4). Section V is devoted to our conclusions. Finally, the appendices collect some technical details.

\section{PRELIMINARIES}

In this section we summarize the basic nonequilibrium features of quantum systems of the type shown in Fig. 1 . Throughout the paper we adopt the coordinates $\{(x, i),: x \leqslant$ $0, i=1,2\}$, where $|x|$ denotes the distance from the defect and $i$ labels the lead.

\section{A. Conserved currents and entropy production}

Let us start by fixing the symmetry content. We consider in this paper physical systems in which both the particle number and the total energy are conserved. Accordingly, the correlation functions are invariant under global $U(1)$ transformations and time translations. These symmetries imply the existence of a conserved particle and energy currents $\left(j_{t}, j_{x}\right)$ and $\left(\theta_{t t}, \theta_{x t}\right)$. Local conservation implies

$$
\begin{gathered}
\partial_{t} j_{t}(t, x, i)-\partial_{x} j_{x}(t, x, i)=0, \\
\partial_{t} \theta_{t t}(t, x, i)-\partial_{x} \theta_{x t}(t, x, i)=0 .
\end{gathered}
$$

In order to generate global conserved charges from $j_{t}$ and $\theta_{t t}$, which define the particle number and total energy respectively, one must impose Kirchhoff's rules,

$$
\sum_{i=1}^{2} j_{x}(t, 0, i)=\sum_{i=1}^{2} \theta_{x t}(t, 0, i)=0
$$

which are assumed in what follows.

The total energy of our system has two components: heat energy and chemical energy. Since the chemical energy density is given by $\mu_{i} j_{t}(t, x, i)$, for the heat density one has [52]

$$
q_{t}(t, x, i)=\theta_{t t}(t, x, i)-\mu_{i} j_{t}(t, x, i) .
$$

Accordingly, the heat current reads

$$
q_{x}(t, x, i)=\theta_{x t}(t, x, i)-\mu_{i} j_{x}(t, x, i) .
$$

Following [52], we introduce at this point the entropy production operator $[3,7]$

$$
\dot{S}(t, x)=-\sum_{i=1}^{2} \beta_{i} q_{x}(t, x, i) .
$$

The definition (11) involves the nonequilibrium heat currents flowing in the leads $L_{i}$ and the equilibrium temperatures $\beta_{i}$ of the heat reservoirs. The operator (11) will be the main subject of our investigation below.

A simple but deep difference between the heat current $q_{x}(t, x, i)$ and entropy production operator $\dot{S}(t, x)$ is worth 
stressing. The current $q_{x}(t, x, i)$ is a local observable, which depends on the lead $L_{i}$ where it is observed or measured. The entropy production operator $\dot{S}(t, x)$ concerns instead the whole system and does not refer to a single lead. Accordingly, the correlation functions (1), which describe the entropy production fluctuations, take into account all the interference effects between the heat currents in the two different leads $L_{1}$ and $L_{2}$. The contribution of the interference terms to (1) is fundamental for proving the bound (4).

It is instructive at this stage to describe the basic physical process taking place in the system in Fig. 1 and generating the entropy production. The conservation laws (6) and (7) obviously imply the local heat current conservation:

$$
\partial_{t} q_{t}(t, x, i)-\partial_{x} q_{x}(t, x, i)=0 .
$$

However, if $\mu_{1} \neq \mu_{2}$ the heat current violates the Kirchhoff rule. One has in fact

$$
\sum_{i=1}^{2} q_{x}(t, 0, i)=\left(\mu_{1}-\mu_{2}\right) j_{x}(t, 0,1) .
$$

Since the total energy is conserved, both the heat and chemical energies are in general not separately conserved. Therefore, for $\mu_{1} \neq \mu_{2}$ the junction in Fig. 1 operates as energy converter without dissipation [53]. The two possible regimes are controlled by the expectation value of the operator

$$
\dot{Q}=-\sum_{i=1}^{2} q_{x}(t, x, i)
$$

in the underlying nonequilibrium state. If $\langle\dot{Q}\rangle<0$ the junction transforms heat to chemical energy. The opposite process takes place if instead $\langle\dot{Q}\rangle>0$. A detailed study of this phenomenon of energy transmutation in the LB state $\Omega_{\mathrm{LB}}$ has been performed in Ref. [53].

The above general considerations apply to the system in Fig. 1 with any dynamics preserving the particle number and total energy. In this sense they are universal. For concretely evaluating the quantum fluctuations associated with $\dot{S}$, one should fix the dynamics and the nonequilibrium state. This is done in the next subsection.

\section{B. Dynamics and the LB state: The Schrödinger junction}

Nonequilibrium systems of the type in Fig. 1 behave in a complicated way, and the linear response or other approximations are usually not enough for fully describing their complexity. For this reason the existence of models, which incorporate the main nonequilibrium features, while being sufficiently simple to be analyzed exactly, is conceptually very important. One such example is provided by particles, which are freely moving along the leads and interact only in the junction $x=0$. This hypothesis accounts remarkably well [54] for the experimental results [55] about the noise in mesoscopic conductors and has been recently confirmed [56] even in the case of fractional charge transport in quantum Hall samples. At the theoretical side, our previous analysis in Refs. [41,42,50,53] shows that this setup represents an exceptional testing ground for exploring general ideas about quantum transport.
One concrete realization of the above scenario is the Schrödinger junction, where the dynamics along the leads is fixed by (the natural units $\hbar=c=k_{\mathrm{B}}=1$ are adopted throughout the paper)

$$
\left(\mathrm{i} \partial_{t}+\frac{1}{2 m} \partial_{x}^{2}\right) \psi(t, x, i)=0,
$$

supplemented by the standard equal-time canonical anticommutation relations. The junction represents physically a pointlike defect localized at $x=0$. The associated interaction determines the scattering matrix $\mathbb{S}$, which is fixed by requiring that the bulk Hamiltonian $-\partial_{x}^{2} / 2 m$ admits a self-adjoint extension in $x=0$. All such extensions are defined [57-59] by the boundary condition

$$
\lim _{x \rightarrow 0^{-}} \sum_{j=1}^{2}\left[\lambda(\mathbb{I}-\mathbb{U})_{i j}+\mathrm{i}(\mathbb{I}+\mathbb{U})_{i j} \partial_{x}\right] \psi(t, x, j)=0,
$$

where $\mathbb{I}$ is the identity matrix, $\mathbb{U}$ is a generic $2 \times 2$ unitary matrix, and $\lambda>0$ is a parameter with dimension of mass. Equation (16) guaranties unitary time evolution and implies [57-59] the scattering matrix

$$
\mathbb{S}(k)=-\frac{[\lambda(\mathbb{I}-\mathbb{U})-k(\mathbb{I}+\mathbb{U})]}{[\lambda(\mathbb{I}-\mathbb{U})+k(\mathbb{I}+\mathbb{U})]},
$$

$k$ being the particle momentum. Equation (17) defines a meromorphic function in the complex $k$-plane.

Since scale invariance preserves the universal features of one-dimensional quantum transport [60] and leads at the same time to relevant simplifications, it is instructive to characterise explicitly the scale-invariant elements in the family (17). For this purpose we first diagonalize $\mathbb{U}$ :

$$
\mathcal{U}^{*} \mathbb{U} \mathcal{U}=\mathbb{U}_{\mathrm{d}}=\operatorname{diag}\left(e^{-2 i \alpha_{1}}, e^{-2 i \alpha_{2}}\right), \quad-\frac{\pi}{2}<\alpha_{i} \leqslant \frac{\pi}{2},
$$

where $*$ stands for Hermitian conjugation. It follows from (17) that the unitary matrix $\mathcal{U}$ diagonalizes $\mathbb{S}(k)$ for any $k$ as well. In fact,

$$
\mathbb{S}_{\mathrm{d}}(k)=\mathcal{U}^{*} \mathbb{S}(k) \mathcal{U}=\operatorname{diag}\left(\frac{k+i \eta_{1}}{k-i \eta_{1}}, \frac{k+i \eta_{2}}{k-i \eta_{2}}\right),
$$

where

$$
\eta_{i} \equiv \lambda \tan \left(\alpha_{i}\right) .
$$

At this point scale invariance implies $[29,61]$ the following alternative:

$$
\eta_{i}=\left\{\begin{array}{lll}
0 & \left(\alpha_{i}=0\right), & \text { Neumann b.c. } \\
\infty & \left(\alpha_{i}=\pi / 2\right), & \text { Dirichlet b.c. }
\end{array}\right.
$$

Accordingly, the scale-invariant scattering matrices, called also critical points, are $k$-independent and are given by the family

$$
\mathbb{S}=\mathcal{U} \mathbb{S}_{\mathrm{d}} \mathcal{U}^{*}, \quad \mathcal{U} \in U(2), \quad \mathbb{S}_{\mathrm{d}}=\operatorname{diag}(1,-1),
$$

supplemented by the two isolated elements $\mathbb{S}= \pm \mathbb{I}$. The latter are not interesting because there is no transmission between the two leads and the system is therefore in equilibrium. We adopt (22) in Sec. III A for deriving the mean value $\langle\dot{S}(t, x)\rangle_{\mathrm{LB}}$ at criticality in explicit form. 
The scattering states associated to (17) read [62]

$$
\chi(k ; x)=\left[\mathrm{e}^{-\mathrm{i} k x} \mathbb{I}+\mathrm{e}^{\mathrm{i} k x} \mathbb{S}^{*}(k)\right], \quad k \geqslant 0 .
$$

Postponing the discussion of the general case, let us assume for the moment that $\mathbb{S}(k)$ has no bound states. Then, the solution of the quantum boundary value problem (15) and (16) is given by

$$
\psi(t, x, i)=\sum_{j=1}^{2} \int_{0}^{\infty} \frac{d k}{2 \pi} \mathrm{e}^{-\mathrm{i} \omega(k) t} \chi_{i j}(k ; x) a_{j}(k),
$$

where $\omega(k)=k^{2} / 2 m$ is the dispersion relation and the operators $\left\{a_{i}(k), a_{i}^{*}(k): k \geqslant 0, i=1,2\right\}$ generate a standard anticommutation relation algebra $\mathcal{A}_{+}$.

Both (15) and (16) are invariant under global $U(1)$ phase transformations and time translations. The relative conserved particle and energy currents have the well-known form

$$
j_{x}(t, x, i)=\frac{\mathrm{i}}{2 m}\left[\psi^{*}\left(\partial_{x} \psi\right)-\left(\partial_{x} \psi^{*}\right) \psi\right](t, x, i)
$$

and

$$
\begin{aligned}
\theta_{x t}(t, x, i)= & \frac{1}{4 m}\left[\left(\partial_{t} \psi^{*}\right)\left(\partial_{x} \psi\right)+\left(\partial_{x} \psi^{*}\right)\left(\partial_{t} \psi\right)\right. \\
& \left.-\left(\partial_{t} \partial_{x} \psi^{*}\right) \psi-\psi^{*}\left(\partial_{t} \partial_{x} \psi\right)\right](t, x, i),
\end{aligned}
$$

respectively. Plugging the solution (24) in (25) and (26), one can express the heat current (10) and therefore the entropy production field operator in terms of the generators of $\mathcal{A}_{+}$. The result is

$$
\begin{aligned}
\dot{S}(t, x)= & \frac{\mathrm{i}}{4 m} \int_{0}^{\infty} \frac{\mathrm{d} k}{2 \pi} \int_{0}^{\infty} \frac{d p}{2 \pi} \mathrm{e}^{\mathrm{i} t[\omega(k)-\omega(p)]} \\
& \times \sum_{l, j=1}^{2} a_{l}^{*}(k) \sum_{i=1}^{2} \beta_{i}\left[2 \mu_{i}-\omega(k)-\omega(p)\right] \\
& \times\left\{\chi_{l i}^{*}(k ; x)\left[\partial_{x} \chi_{i j}\right](p ; x)\right. \\
& \left.-\left[\partial_{x} \chi_{l i}^{*}\right](k ; x) \chi_{i j}(p ; x)\right\} a_{j}(p) .
\end{aligned}
$$

This equation defines $\dot{S}(t, x)$ as a quadratic element of the algebra $\mathcal{A}_{+}$. In order to extract the physical information we are interested in, one must fix a representation of $\mathcal{A}_{+}$. The physical setup in Fig. 1 suggests adopting the LB representation of $\mathcal{A}_{+}$, which generalizes the equilibrium Gibbs representation to the case of systems driven away from equilibrium by a particle and energy exchange with more then one heat reservoir. A field theoretical construction of the Hilbert space $\left\{\mathcal{H}_{\mathrm{LB}},(\cdot, \cdot)\right\}$ of this representation is given in Ref. [62]. For deriving the expectation values of (27) one can concentrate on the $2 n$-point function

$$
\begin{gathered}
\left(\Omega_{\mathrm{LB}}, a_{l_{1}}^{*}\left(k_{1}\right) a_{m_{1}}\left(p_{1}\right) \cdots a_{l_{n}}^{*}\left(k_{n}\right) a_{m_{n}}\left(p_{n}\right) \Omega_{\mathrm{LB}}\right) \\
\equiv\left\langle a_{l_{1}}^{*}\left(k_{1}\right) a_{m_{1}}\left(p_{1}\right) \cdots a_{l_{n}}^{*}\left(k_{n}\right) a_{m_{n}}\left(p_{n}\right)\right\rangle_{\mathrm{LB}},
\end{gathered}
$$

which can be represented as a kind of Slater determinant, whose explicit form (A3) is given in Appendix A. Using (A3) we derive in what follows the correlation functions of the operator $\dot{S}$ in the LB representation $\mathcal{H}_{\mathrm{LB}}$ and discuss the physical implications.

\section{ENTROPY PRODUCTION CORRELATION FUNCTIONS}

\section{A. The one-point function}

It is natural to start with the one point function $\langle\dot{S}(t, x)\rangle_{\mathrm{LB}}$, which gives the mean value of the entropy production in the LB state $\Omega_{\mathrm{LB}}$. Using (27) and (A3) for $n=1$, one easily obtains the integral representation (5) with

$$
\mathcal{M}_{1}(\omega)=\tau(\omega)\left[\gamma_{2}(\omega)-\gamma_{1}(\omega)\right]\left[d_{1}(\omega)-d_{2}(\omega)\right] .
$$

Here

$$
\tau(\omega)=\left|\mathbb{S}_{12}(\sqrt{2 m \omega})\right|^{2}
$$

is the transmission probability

$$
\gamma_{i}(\omega)=\beta_{i}\left(\omega-\mu_{i}\right), \quad i=1,2,
$$

and $d_{i}(\omega)$ is the Fermi distribution

$$
d_{i}(\omega)=\frac{1}{1+\mathrm{e}^{\gamma_{i}(\omega)}}
$$

of the reservoir $R_{i}$. One can easily check now that both square brackets $[\cdots]$ of (29) have always the same sign or vanish simultaneously. Therefore,

$$
\mathcal{M}_{1}(\omega) \geqslant 0
$$

which proves (4) for $n=1$. In addition, $\mathcal{M}_{1}(\omega)=0$ for any $\omega$ implies the equilibrium regime $\beta_{1}=\beta_{2}$ and $\mu_{1}=\mu_{2}$.

It is worth mentioning that $\langle\dot{S}(t, x)\rangle_{\mathrm{LB}}$, given by (5) and (29), is both time and position independent. The $t$ independence follows from the energy conservation, whereas the $x$-independence is a consequence of the heat current conservation (12). Clearly these are peculiar properties of the one-point function $w_{1}[\dot{S}]$. The study of $\left\{w_{n}[\dot{S}],: n \geqslant 2\right\}$ in the next subsection reveals a more complicated behavior.

Let us explore in conclusion the scale-invariant regime. At criticality the transmission probability $\tau$ is constant and plugging (29) in (5) one can perform the $\omega$-integration explicitly. The result is

$$
\begin{aligned}
\langle\dot{S}(t, x)\rangle_{\mathrm{LB}}= & \left(\lambda_{2}-\lambda_{1}\right) \frac{\tau}{2 \pi}\left[\frac{1}{\beta_{2}} \ln \left(1+\mathrm{e}^{\lambda_{2}}\right)-\frac{1}{\beta_{1}} \ln \left(1+\mathrm{e}^{\lambda_{1}}\right)\right] \\
& +\left(\beta_{1}-\beta_{2}\right) \frac{\tau}{2 \pi}\left[\frac{1}{\beta_{1}^{2}} \mathrm{Li}_{2}\left(-\mathrm{e}^{\lambda_{1}}\right)-\frac{1}{\beta_{2}^{2}} \mathrm{Li}_{2}\left(-\mathrm{e}^{\lambda_{2}}\right)\right],
\end{aligned}
$$

where $\lambda_{i} \equiv \beta_{i} \mu_{i}$ are dimensionless parameters and $\mathrm{Li}_{2}$ is the dilogarithm function.

The mean value the entropy production (34) is generated by both the temperature and the chemical potential differences of the heat reservoirs. In order to get an idea about the separate effect of these two independent sources, it is instructive to consider the limiting regimes $\beta_{1}=\beta_{2}, \mu_{1} \neq \mu_{2}$ on one hand and $\beta_{1} \neq \beta_{2}, \mu_{1}=\mu_{2}$ on the other. These ranges of parameters are interesting also from the experimental point of view.

Let us assume first that that the heat reservoirs have the same temperature $\beta_{1}=\beta_{2}=\beta$. In this regime the dilogarithms in (34) do not contribute, and at high temperature one finds

$$
\lim _{\beta \rightarrow 0}\langle\dot{S}(t, x)\rangle_{\mathrm{LB}}^{\beta_{1}=\beta_{2}}=0 .
$$




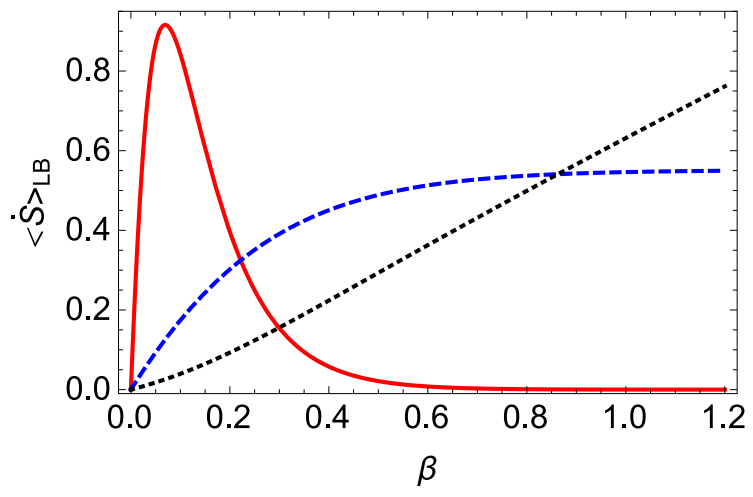

FIG. 2. Entropy production (in temperature units) for $\beta_{1}=\beta_{2}=$ $\beta$ and $\tau=1$ with $\left(\mu_{1}, \mu_{2}\right)=(-30,-10)$ (red continuous line), $(-5,0)$ (blue dashed line), and $(4,6)$ (black dotted line).

The behavior at low temperature depends on $\mu_{i}$. Observing that $\langle\dot{S}(t, x)\rangle_{\mathrm{LB}}^{\beta_{1}=\beta_{2}}$ is a symmetric function of $\left(\mu_{1}, \mu_{2}\right)$, one can assume without loss of generality that $\mu_{1}<\mu_{2}$ and obtain

$$
\lim _{\beta \rightarrow \infty}\langle\dot{S}(t, x)\rangle_{\mathrm{LB}}^{\beta_{1}=\beta_{2}}= \begin{cases}0, & \text { for } \mu_{2}<0, \\ \frac{-\mu_{1} \tau \ln 2}{2 \pi}, & \text { for } \mu_{2}=0, \\ \infty, & \text { for } \mu_{2}>0\end{cases}
$$

as shown in Fig. 2.

In the second case we set $\mu_{1}=\mu_{2}=\mu$. The origin of the entropy increase is therefore exclusively the difference between the temperatures $\beta_{1} \neq \beta_{2}$ of the two heat reservoirs. In this case the dilogarithms in (34) have a relevant contribution, $\langle\dot{S}(t, x)\rangle_{\mathrm{LB}}^{\mu_{1}=\mu_{2}}$ is a symmetric function of $\left(\beta_{1}, \beta_{2}\right)$, and one has

$$
\begin{aligned}
& \lim _{\mu \rightarrow-\infty}\langle\dot{S}(t, x)\rangle_{\mathrm{LB}}^{\mu_{1}=\mu_{2}}=0, \\
& \lim _{\mu \rightarrow \infty}\langle\dot{S}(t, x)\rangle_{\mathrm{LB}}^{\mu_{1}=\mu_{2}}=\frac{\pi\left(\beta_{1}-\beta_{2}\right)^{2}\left(\beta_{1}+\beta_{2}\right) \tau}{12 \beta_{1}^{2} \beta_{2}^{2}},
\end{aligned}
$$

as displayed in Fig. 3.

Finally, for $\tau=1$ the defect at $x=0$ is absent and one obtains from (34) the mean entropy production of two heat reservoirs connected with a homogeneous infinite lead.

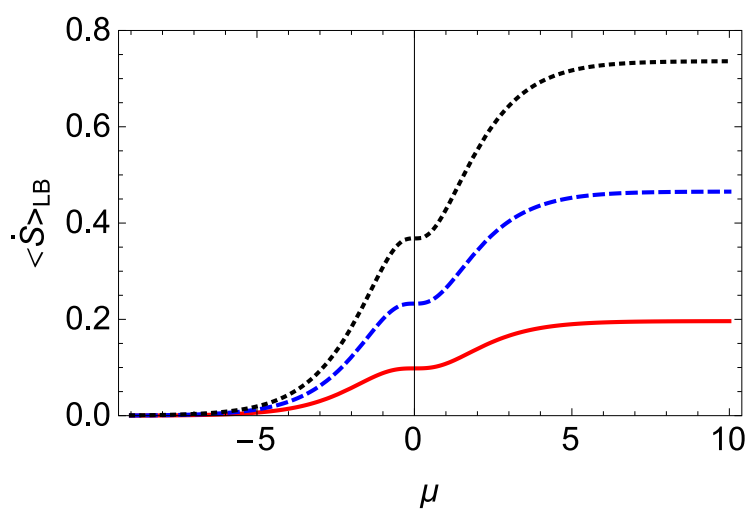

FIG. 3. Entropy production (in temperature units) for $\mu_{1}=\mu_{2}=$ $\mu$ and $\tau=1$ with $\left(\beta_{1}, \beta_{2}\right)=(1,2)$ (red continuous line), (1,3) (blue dashed line), and $(1,4)$ (black dotted line).

\section{B. The $\boldsymbol{n}$-point function}

First of all we observe that the correlation function $w_{n}[\dot{S}]$ depends on the time differences

$$
\hat{t}_{k} \equiv t_{k}-t_{k+1}, \quad k=1, \ldots, n-1,
$$

which is a consequence of the time translation invariance of $\Omega_{\mathrm{LB}}$. Since the defect at $x=0$ violates translation invariance in space, $w_{n}[\dot{S}]$ depends on all the coordinates $\left\{x_{l}: l=\right.$ $1, \ldots, n\}$ separately. In order to simplify the analysis and avoid those variables, which are marginal for the entropy production, we introduce the Fourier transforms

$$
\begin{aligned}
& \mathcal{W}_{n}[\dot{S}]\left(x_{1}, \ldots, x_{n} ; v\right)=\int_{-\infty}^{\infty} d \hat{t}_{1} \ldots \\
& \int_{-\infty}^{\infty} d \hat{t}_{n-1} \mathrm{e}^{-\mathrm{i} v\left(\hat{t}_{1}+\cdots \hat{t}_{n-1}\right)} w_{n}[\dot{S}]\left(t_{1}, x_{1}, \ldots, t_{n}, x_{n}\right), \quad n \geqslant 2,
\end{aligned}
$$

and perform the zero-frequency limit

$$
\mathcal{W}_{n}[\dot{S}]=\lim _{\nu \rightarrow 0^{+}} \mathcal{W}_{n}[\dot{S}]\left(x_{1}, \ldots, x_{n} ; \nu\right)
$$

This limit has been adopted already in the classical studies [37-41] of quantum noise produced by the particle current for $n=2$. It has been extended in Ref. [48] to the current cumulants with $n>2$ and applied in the framework of full counting statistics [43-50] as well. The zero frequency regime has a well known physical meaning and is mostly explored in experiments. As mentioned in the introduction, in the range of low frequencies all quantum fluctuation are integrated over long period of time. It is evident from (39) that in the limit $v \rightarrow 0$ this period becomes actually the whole line. We show in Appendix B that the structure of $w_{n}[\dot{S}]$ greatly simplifies in this case. In fact, using the definition (27) of $\dot{S}$ and the correlation function (A3), one finds

$$
\mathcal{W}_{n}[\dot{S}]=\int_{0}^{\infty} \frac{d \omega}{2 \pi}\left[\gamma_{2}(\omega)-\gamma_{1}(\omega)\right]^{n} \mathbb{D}_{n}(\omega) .
$$

The basic steps in deriving the representation (41), as well as the explicit form (B2) of the factor $\mathbb{D}_{n}(\omega)$ in the integrand, are given in Appendix B. $\mathbb{D}_{n}(\omega)$ is a sum of determinants, which depend on the scattering matrix (17) and the Fermi distribution (32), in other words on $\tau(\omega)$ and $\left(\beta_{i}, \mu_{i}\right)$. It has been shown in Ref. [42] that the bound states of $\mathbb{S}$, if they exist, do not contribute in the limit (40) as well. Despite these significant simplifications, at the first sight the integrand of (41) for generic $n$ might look still complicated. As shown in Appendix B, however, this is not the case, and the final expression reads

$$
\mathcal{W}_{n}[\dot{S}]=\int_{0}^{\infty} \frac{d \omega}{2 \pi} \mathcal{M}_{n}(\omega),
$$

with

$$
\begin{aligned}
\mathcal{M}_{2 k-1} & =\tau^{k}\left(\gamma_{2}-\gamma_{1}\right)^{2 k-1} c_{1}, \\
\mathcal{M}_{2 k} & =\tau^{k}\left(\gamma_{2}-\gamma_{1}\right)^{2 k} c_{2} .
\end{aligned}
$$


Here $k=1,2, \ldots$, the $\omega$-dependence of all factors has been suppressed for conciseness, and the combinations

$$
c_{1} \equiv d_{1}-d_{2}, \quad c_{2} \equiv d_{1}+d_{2}-2 d_{1} d_{2}
$$

have been introduced for convenience.

The explicit form (43) and (44) of the integrands $\mathcal{M}_{n}$ represents a key point of our analysis of the fluctuations of the entropy production. First, from (43) and (44) one infers the result (4) announced in the introduction, namely, that all $\mathcal{M}_{n}$ are non-negative. In fact, the argument about the positivity of $\mathcal{M}_{1}$ applies actually for all odd values of $n$. The inequality (44) for even values of $n$ follows instead from

$$
c_{2}=\frac{\mathrm{e}^{\gamma_{1}}+\mathrm{e}^{\gamma_{2}}}{\left(1+\mathrm{e}^{\gamma_{1}}\right)\left(1+\mathrm{e}^{\gamma_{2}}\right)} \geqslant 0 .
$$

Our goal in the next section will be to show that the integrands (43) and (44) represent indeed the moments of a probability distribution and to reconstruct this distribution.

\section{PROBABILITY DISTRIBUTION GOVERNING THE ENTROPY PRODUCTION}

The fluctuations of a quantum observable give rise in general to a quasiprobability distribution. Familiar examples are the Wigner function [63], some distributions stemming from coherent states in quantum optics [64,65], and more recent examples associated with time-integrated observables $[66,67]$ in the context of full quantum statistics [43-47]. In this section we show that $\dot{S}$ generates in the LB state $\Omega_{\mathrm{LB}}$ a true probability distribution $\varrho$. The idea is to reconstruct $\varrho$ from the moments (43) and (44), solving the underlying moment problem.

\section{A. Solution of the moment problem}

We are looking for a function $\varrho$ with domain $\mathcal{D}$ such that

$$
\mathcal{M}_{n}=\int_{\mathcal{D}} d \sigma \sigma^{n} \varrho(\sigma), \quad n=0,1, \ldots,
$$

where $\mathcal{M}_{n}$ are given for $n \geqslant 1$ by (43) and (44), and

$$
\mathcal{M}_{0}=1
$$

provides a normalization condition. The parameter $\sigma$ describes the entropy production. There exist [68] three possible choices for the domain $\mathcal{D}$ of $\sigma$ : the whole line $\mathcal{D}=\mathbb{R}$, the half line $\mathcal{D}=\mathbb{R}_{+}$, and a compact interval $\mathcal{D}=[a, b]$. In order to determine $\mathcal{D}$ we have to investigate the Hankel determinants

$$
\mathbb{H}_{n} \equiv\left|\begin{array}{cccc}
\mathcal{M}_{0} & \mathcal{M}_{1} & \ldots & \mathcal{M}_{n} \\
\mathcal{M}_{1} & \mathcal{M}_{2} & \ldots & \mathcal{M}_{n+1} \\
\vdots & \vdots & \vdots & \vdots \\
\mathcal{M}_{n} & \mathcal{M}_{n+1} & \cdots & \mathcal{M}_{2 n}
\end{array}\right|
$$

A necessary and sufficient condition for the existence of $\varrho$ on $\mathbb{R}$ is [68]

$$
\mathbb{H}_{n} \geqslant 0, \quad \forall n=1,2, \ldots
$$

Using (43), (44), and (48) one gets

$$
\begin{aligned}
& \mathbb{H}_{0}=1, \quad \mathbb{H}_{1}=\tau\left(\gamma_{1}-\gamma_{2}\right)^{2}\left(c_{2}-\tau c_{1}^{2}\right), \\
& \mathbb{H}_{2}=\tau^{3}\left(\gamma_{1}-\gamma_{2}\right)^{6}\left(1-c_{2}\right)\left(c_{2}^{2}-\tau c_{1}^{2}\right), \quad \mathbb{H}_{n \geqslant 3}=0 .
\end{aligned}
$$

Combining the inequalities

$$
0 \leqslant c_{2} \leqslant 1, \quad c_{2}^{2}-c_{1}^{2} \geqslant 0,
$$

which follow directly from the explicit form (45) of $c_{i}$ and using $0 \leqslant \tau \leqslant 1$, one gets that both $\mathbb{H}_{1}$ and $\mathbb{H}_{2}$ are non-negative. Since in addition,

$$
\mathbb{H}_{2}^{\prime} \equiv\left|\begin{array}{ll}
\mathcal{M}_{1} & \mathcal{M}_{2} \\
\mathcal{M}_{2} & \mathcal{M}_{3}
\end{array}\right|=\tau^{2}\left(\gamma_{1}-\gamma_{2}\right)^{4}\left(c_{1}^{2} \tau-c_{2}^{2}\right) \leqslant 0,
$$

the domains $\mathbb{R}_{+}$and $[a, b]$ are excluded [68].

Summarizing, the entropy production $\sigma$ in the LB state $\Omega_{\mathrm{LB}}$ gives rise to the so-called Hamburger moment problem $\mathcal{D}=\mathbb{R}$. Moreover, since $\mathbb{H}_{n \geqslant 3}=0$ the general theory [68] implies that $\varrho$ is fully localized at three different values of $\sigma$.

Once the domain $\mathcal{D}$ has been determined, the explicit form of the distribution $\varrho$ can be recovered [68] by performing the Fourier transform

$$
\varrho(\sigma)=\int_{-\infty}^{\infty} \frac{d \lambda}{2 \pi} \mathrm{e}^{-\mathrm{i} \lambda \sigma} \varphi(\lambda)
$$

of the generating function

$$
\varphi(\lambda)=\sum_{n=0}^{\infty} \frac{(\mathrm{i} \lambda)^{n}}{n !} \mathcal{M}_{n} .
$$

Employing (43), (44), (48) one finds

$$
\begin{aligned}
\varphi(\lambda)= & 1+\mathrm{i} c_{1} \sqrt{\tau} \sin \left[\lambda\left(\gamma_{2}-\gamma_{1}\right) \sqrt{\tau}\right] \\
& +c_{2}\left\{\cos \left[\lambda\left(\gamma_{2}-\gamma_{1}\right) \sqrt{\tau}\right]-1\right\},
\end{aligned}
$$

whose Fourier transform reads

$$
\begin{aligned}
\varrho(\sigma)= & \frac{1}{2}\left(c_{2}-c_{1} \sqrt{\tau}\right) \delta\left[\sigma-\left(\gamma_{1}-\gamma_{2}\right) \sqrt{\tau}\right]+\left(1-c_{2}\right) \delta(\sigma) \\
& +\frac{1}{2}\left(c_{2}+c_{1} \sqrt{\tau}\right) \delta\left[\sigma-\left(\gamma_{2}-\gamma_{1}\right) \sqrt{\tau}\right] .
\end{aligned}
$$

Equation (58) confirms that the entropy production is indeed localized in three points on the $\sigma$-line. It is convenient to adopt at this stage the variables $\sigma_{i j}$ defined by (3), which read

$$
\sigma_{i j}=\left(\gamma_{i}-\gamma_{j}\right) \sqrt{\tau}
$$

in terms of $\gamma_{i}$ and $\tau$. Then $\varrho$ can be rewritten the form

$$
\varrho(\sigma)=p_{12} \delta\left(\sigma-\sigma_{12}\right)+p \delta(\sigma)+p_{21} \delta\left(\sigma-\sigma_{21}\right)
$$

with

$$
p_{12}=\frac{1}{2}\left(c_{2}-c_{1} \sqrt{\tau}\right), \quad p_{21}=\frac{1}{2}\left(c_{2}+c_{1} \sqrt{\tau}\right), \quad p=1-c_{2} .
$$

Here $p_{i j}$ is the probability of emission of a particle by the reservoir $R_{i}$ and absorption by $R_{j}$, whereas $p$ is the probability for emission and absorption by the same reservoir $R_{1}$ or $R_{2}$. One can easily show in fact that

$$
p_{12}+p+p_{21}=1, \quad p_{i j} \in[0,1], \quad p \in[0,1],
$$

implying that $\varrho$ is a true probability distribution. 


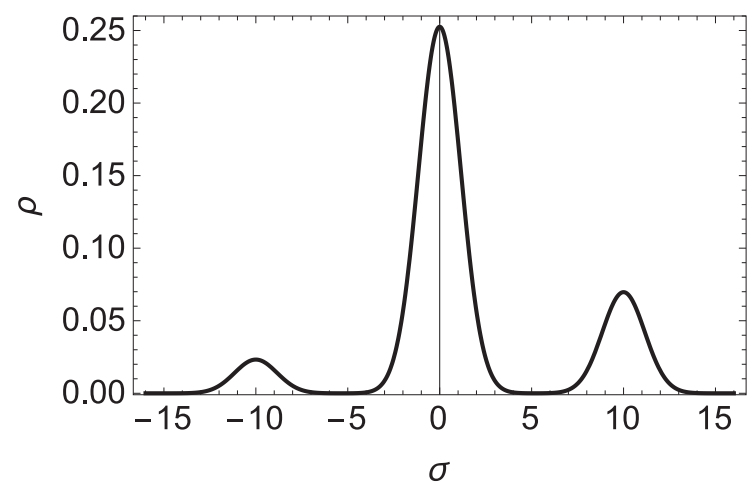

FIG. 4. The smeared distribution $\varrho_{v}$ with $v=2 / 3, \gamma_{1}=21, \gamma_{2}=$ 1 and $\tau=1 / 4$.

It is worth stressing that the probabilities (62) refer to arbitrary but fixed energy $\omega \in[0, \infty)$. At this energy the probabilities for $n$-particle emission and absorption with $n \geqslant 2$ vanish because of Pauli's principle. This is not the case for the bosonic junctions discussed in [51], where multiparticle emission and absorption processes with the same energy are allowed.

As anticipated in the introduction, we have shown that both processes with positive and negative entropy production appear at the quantum level. It is quite intuitive that if the transport of a particle from the red to the blue reservoir in the isolated system in Fig. 1 increases the entropy, the opposite process is decreasing it. The crucial point is that according to (61) both these events have a nonvanishing probability and are present without invoking any time reversal operation.

Since $\varrho$ is not smooth but a generalized function, in order to illustrate graphically its behavior it is convenient to introduce the $\delta$-sequence

$$
\delta_{v}(\sigma)=\frac{v}{\sqrt{\pi}} \mathrm{e}^{-v^{2} \sigma^{2}}, \quad v>0
$$

and consider the smeared distribution

$$
\varrho_{v}(\sigma)=p_{12} \delta_{\nu}\left(\sigma-\sigma_{12}\right)+p \delta_{v}(\sigma)+p_{21} \delta_{v}\left(\sigma-\sigma_{21}\right) .
$$

As is well known, for $v \rightarrow \infty$ one has $\varrho_{v} \rightarrow \varrho$ in the sense of generalized functions. The plots of $\varrho_{v}$ for finite values of $v$ nicely illustrate the physics behind the distribution $\varrho$. One example is reported in Fig. 4. The shape of $\varrho_{\nu}$ depends on $v$, but the events with positive entropy production always dominate those with negative one. This feature is a consequence of the property

$$
\sigma_{i j}>0 \Longrightarrow p_{i j}>p_{j i}
$$

which is $v$-independent and holds therefore also in the limit $v \rightarrow \infty$.

It is instructive in this point to derive the ratio $P_{+} / P_{-}$where $P_{+}$and $P_{-}$are the probabilities to have positive and negative entropy production respectively. Without loss of generality one can assume for this purpose that $\sigma_{12}>0$. Then

$$
\begin{aligned}
\frac{P_{+}}{P_{-}} & =\frac{p_{12}}{p_{21}}=\frac{c_{2}-c_{1} \sqrt{\tau}}{c_{2}+c_{1} \sqrt{\tau}} \\
& =\frac{(1-\sqrt{\tau})+(1+\sqrt{\tau}) \mathrm{e}^{\sigma_{12} / \sqrt{\tau}}}{(1+\sqrt{\tau})+(1-\sqrt{\tau}) \mathrm{e}^{\sigma_{12} / \sqrt{\tau}}} .
\end{aligned}
$$

Equation (66) generalizes the fluctuation relation, discussed in Refs. [17-23], to the case in which space translation invariance is broken by a quantum pointlike defect with transmission probability $\tau$. In the limit $\tau \rightarrow 1$ the defect disappears, the system becomes homogeneous, and one recovers from (66) the result of Crooks [17],

$$
\lim _{\tau \rightarrow 1} \frac{P_{+}}{P_{-}}=\mathrm{e}^{\sigma_{12}},
$$

originally obtained in the context of stochastic dynamics.

Summarizing, the probability distribution (58) fully describes the entropy production zero-frequency fluctuations in the LB state $\Omega_{\mathrm{LB}}$. It is natural to expect that the behavior of $\varrho$ depends on the choice of this state. This expectation is confirmed in the next subsection, where the $\dot{S}$ fluctuations in the state generated from $\Omega_{\mathrm{LB}}$ by time reversal are explored.

\section{B. Impact of time reversal}

As before, we consider the field $\psi$ defined by (24) in the LB representation $\left\{\mathcal{H}_{\mathrm{LB}},(\cdot, \cdot)\right\}$ of the algebra $\mathcal{A}_{+}$. The time reversal operation acts as usual,

$$
T \psi(t, x, i) T^{-1}=\eta_{T} \psi(-t, x, i),
$$

where $\left|\eta_{T}\right|=1$ and $T$ is an antiunitary operator in $\mathcal{H}_{\mathrm{LB}}$ with $T^{2}=\mathbb{I}$. Using (25) and (26) one easily gets

$$
\begin{gathered}
T j_{x}(t, x, i) T^{-1}=-j_{x}(-t, x, i), \\
T \theta_{t x}(t, x, i) T^{-1}=-\theta_{t x}(-t, x, i) .
\end{gathered}
$$

Since $\left\langle j_{x}(t, x, i)\right\rangle_{\mathrm{LB}} \neq 0$ and $\left\langle\theta_{t x}(t, x, i)\right\rangle_{\mathrm{LB}} \neq 0$, the overall minus signs in the right-hand side of (69) and (70) imply that $T \Omega_{\mathrm{LB}} \neq \Omega_{\mathrm{LB}}$. Therefore, $T$ generates another state $\Omega_{\mathrm{LB}}^{T}=$ $T \Omega_{\mathrm{LB}} \in \mathcal{H}_{\mathrm{LB}}$ of the system. The entropy fluctuations in this new state are described by

$$
\begin{aligned}
w_{n}^{T}[\dot{S}]\left(t_{1}, x_{1}, \ldots, t_{n}, x_{n}\right) & =\left\langle\dot{S}\left(t_{1}, x_{1}\right) \cdots \dot{S}\left(t_{n}, x_{n}\right)\right\rangle_{\mathrm{LB}}^{T} \\
& \equiv\left(T \Omega_{\mathrm{LB}}, \dot{S}\left(t_{1}, x_{1}\right) \cdots \dot{S}\left(t_{n}, x_{n}\right) T \Omega_{\mathrm{LB}}\right),
\end{aligned}
$$

where $(\cdot, \cdot)$ is the scalar product in $\mathcal{H}_{\mathrm{LB}}$. Using (69) and (70) one finds that

$$
w_{2 k-1}^{T}[\dot{S}]=-w_{2 k-1}[\dot{S}], \quad w_{2 k}^{T}[\dot{S}]=w_{2 k}[\dot{S}],
$$

with $k=1,2, \ldots$ Therefore, the momenta $\mathcal{M}_{n}^{T}$ of the probability distribution $\varrho^{T}(\sigma)$ in the time reversed LB state $\Omega_{\mathrm{LB}}^{T}$ satisfy

$$
\mathcal{M}_{2 k-1}^{T} \leqslant 0, \quad \mathcal{M}_{2 k}^{T} \geqslant 0,
$$

which is the mathematical consequence of the physical fact that the processes of emission and absorption are inverted with respect to those in $\Omega_{\mathrm{LB}}$. 


\section{Comment}

In the context of particle full counting statistics the possibility to equip the system in Fig. 1 with a measuring device, representing a kind of galvanometer, has been also considered in the literature [46-49]. Following Ref. [46], this alternative scenario can be implemented by introducing in (15) the minimal coupling $\mathrm{i} \partial_{x} \longmapsto \mathrm{i} \partial_{x}+A(x)$ with the external field $A(x) \sim \delta(x)$. The physical differences between the two setups have been discussed in detail in Ref. [47]. Working out the moments of the entropy production distribution in the presence of a galvanometer, one finds $(k=1,2, \ldots)$

$$
\begin{aligned}
\mathcal{M}_{2 k-1}^{\prime} & =\tau\left(\gamma_{2}-\gamma_{1}\right)^{2 k-1} c_{1}, \\
\mathcal{M}_{2 k}^{\prime} & =\tau\left(\gamma_{2}-\gamma_{1}\right)^{2 k} c_{2},
\end{aligned}
$$

which differ from (43) and (44) only by the power of $\tau$. Since $0 \leqslant \tau \leqslant 1$ one concludes that $\mathcal{M}_{n}^{\prime}$ satisfy the bound (4) as well.

The function, generating (74) and (75), is given by

$$
\begin{aligned}
\varphi^{\prime}(\lambda)= & 1+\mathrm{i} c_{1} \tau \sin \left[\lambda\left(\gamma_{2}-\gamma_{1}\right)\right] \\
& +c_{2} \tau\left\{\cos \left[\lambda\left(\gamma_{2}-\gamma_{1}\right)\right]-1\right\}
\end{aligned}
$$

and leads to the following probability distribution:

$$
\varrho^{\prime}(\sigma)=p_{12}^{\prime} \delta\left(\sigma-\sigma_{12}^{\prime}\right)+p^{\prime} \delta(\sigma)+p_{21}^{\prime} \delta\left(\sigma-\sigma_{21}^{\prime}\right),
$$

with

$$
p_{12}^{\prime}=\frac{\tau}{2}\left(c_{2}-c_{1}\right), \quad p_{21}^{\prime}=\frac{\tau}{2}\left(c_{2}+c_{1}\right), \quad p^{\prime}=1-c_{2} \tau
$$

and

$$
\sigma_{i j}^{\prime}=\left(\gamma_{i}-\gamma_{j}\right)
$$

One can easily verify that (78) satisfy also in this case (62) and define therefore the relative probabilities controlling the particle emission-absorption processes. This feature provides a nice check on the whole setup with a measuring device.

In conclusion, the bound (4) is preserved in the presence of a galvanometer as well.

\section{OUTLOOK AND CONCLUSIONS}

The present paper pursues further the quantum field theory analysis of the physical properties of the LB nonequilibrium steady state. It focuses on the quantum fluctuations of the entropy production in the fermionic system shown in Fig. 1. The junction acts as a nondissipative converter of heat to chemical potential energy and vice versa. During the energy transmutation, particles are emitted and absorbed by the heat reservoirs, which induces a nontrivial entropy production. Processes with positive, vanishing, and negative entropy production occur at the quantum level. In order to characterize the relative impact of these events, we investigate the correlation functions of the entropy production operator in the LB state. The one-point function describes the mean entropy production, whereas the $n$-point functions with $n \geqslant 2$ capture the relative fluctuations. We discover that in the zero frequency limit these fluctuations generate a true probability distribution, whose moments are all positive. Since the first moment describes the mean entropy production, this remarkable property can be interpreted as a kind of extension of the second principle of thermodynamics to the nonequilibrium quantum fluctuations in the LB state. The search for other nonequilibrium states, which share the same entropy production properties with the LB state, is a challenging open problem.

The results of this paper persists even after introducing a galvanometer in the system and can be generalized in several directions. Along the above lines one can study multiterminal systems as well as the Tomonaga-Luttinger liquid away from equilibrium $[69,70]$. The effect of the quantum statistics on the entropy production represents also a deep question, which deserves further study. We are currently investigating [51] this effect in the bosonic version of the fermion system studied above.

\section{ACKNOWLEDGMENTS}

The work of L.S. is supported by the Netherlands Organisation for Scientific Research (NWO). We thank the referees for constructive criticism.

\section{APPENDIX A: CORRELATION FUNCTIONS IN THE LB REPRESENTATION}

In their original work [32,33] Landauer and Büttiker derived the two- and four-point correlation functions of $\left\{a_{i}(k), a_{i}^{*}(k)\right.$ : $k \geqslant 0, i=1,2\}$ in the LB representation $\left\{\mathcal{H}_{\mathrm{LB}},(\cdot, \cdot)\right\}$ using quantum mechanical tools. If one is interested in generic $n$-point functions, it is more convenient to adopt the formalism of second quantisation developed in Ref. [62]. The correlation function (28), needed for the derivation of the entropy production fluctuations, is defined in this formalism by

$$
\begin{aligned}
& \left\langle a_{l_{1}}^{*}\left(k_{1}\right) a_{m_{1}}\left(p_{1}\right) \cdots a_{l_{n}}^{*}\left(k_{n}\right) a_{m_{n}}\left(p_{n}\right)\right\rangle_{\beta, \mu} \\
& \quad=\frac{1}{Z} \operatorname{Tr}\left[\mathrm{e}^{-K} a_{l_{1}}^{*}\left(k_{1}\right) a_{m_{1}}\left(p_{1}\right) \cdots a_{l_{n}}^{*}\left(k_{n}\right) a_{m_{n}}\left(p_{n}\right)\right], \\
& k_{i}>0, p_{i}>0,
\end{aligned}
$$

where

$$
K=\int_{0}^{\infty} \frac{d k}{2 \pi} \sum_{i=1}^{2} \gamma_{i}[\omega(k)] a_{i}^{*}(k) a_{i}(k), \quad Z=\operatorname{Tr}\left(\mathrm{e}^{-K}\right) .
$$

Referring for the details to Refs. [50,62], we report the final result

$$
\begin{aligned}
& \left\langle a_{l_{1}}^{*}\left(k_{1}\right) a_{m_{1}}\left(p_{1}\right) \cdots a_{l_{n}}^{*}\left(k_{n}\right) a_{m_{n}}\left(p_{n}\right)\right\rangle_{\mathrm{LB}} \\
& =\left|\begin{array}{cccc}
\Delta_{l_{1} m_{1}}\left(k_{1}, p_{1}\right) & \Delta_{l_{1} m_{2}}\left(k_{1}, p_{2}\right) & \cdots & \Delta_{l_{1} m_{n}}\left(k_{1}, p_{n}\right) \\
-\widetilde{\Delta}_{l_{2} m_{1}}\left(k_{2}, p_{1}\right) & \Delta_{l_{2} m_{2}}\left(k_{2}, p_{2}\right) & \cdots & \Delta_{l_{2} m_{n}}\left(k_{2}, p_{n}\right) \\
\vdots & \vdots & \vdots & \vdots \\
-\widetilde{\Delta}_{l_{n} m_{1}}\left(k_{n}, p_{1}\right) & -\widetilde{\Delta}_{l_{n} m_{2}}\left(k_{n}, p_{2}\right) & \cdots & \Delta_{l_{n} m_{n}}\left(k_{n}, p_{n}\right)
\end{array}\right| .
\end{aligned}
$$

Here

$$
\begin{aligned}
& \Delta_{l m}(k, p) \equiv 2 \pi \delta(k-p) \delta_{l m} d_{l}[\omega(k)], \\
& \widetilde{\Delta}_{l m}(k, p) \equiv 2 \pi \delta(k-p) \delta_{l m} \widetilde{d}_{l}[\omega(k)],
\end{aligned}
$$


where $d_{l}(\omega)$ is the Fermi distribution (32) and

$$
\tilde{d}_{l}(\omega)=1-d_{l}(\omega)=\frac{\mathrm{e}^{\gamma_{l}(\omega)}}{1+\mathrm{e}^{\gamma_{l}(\omega)}} .
$$

\section{APPENDIX B: DERIVATION OF $\mathbb{D}_{n}$}

We summarize first the main steps in deriving the integral representation (41). Using (27) and (A3) one gets a representation of the correlation function $w_{n}[\dot{S}]\left(t_{1}, x_{1}, \ldots, t_{n}, x_{n}\right)$ which involves $n$ integrations over $k_{i}$ and $n$ integrations over $p_{j}$. Then one proceeds as follows:

(1) By means of the delta functions in ((A4), (A5)) one eliminates all $n$ integrals in $p_{j}$

(2) Plugging the obtained expression in (39), one performs all $(n-1)$ integrals in $\hat{t}_{l}$

(3) At $v=0$ the latter produce $(n-1)$ delta functions, which allow to eliminate all the integrals over $k_{i}$ except one, for instance, that over $k_{1}=k$

(4) Now the curly bracket factor $\{\cdots\}$ in (27) gives the $x$-independent expression

$$
\begin{aligned}
& \mathrm{i}\left\{\chi_{l i}^{*}(k ; x)\left[\partial_{x} \chi_{i j}\right](k ; x)-\left[\partial_{x} \chi_{l i}^{*}\right](k ; x) \chi_{i j}(k ; x)\right\} \\
& \quad=-2 \mathrm{i} k\left[\delta_{l i} \delta_{i j}-\mathbb{S}_{l i}(k) \overline{\mathbb{S}}_{j i}(k)\right],
\end{aligned}
$$

the bar indicating complex conjugation and

(5) Finally, in the integral over $k$ one switches to the variable $\omega=k^{2} / 2 m$.

Following the above steps, one arrives at the integral representation (41) with

$$
\mathbb{D}_{n}=\sum_{i_{1}, \ldots, i_{n}=1}^{2}\left|\begin{array}{cccc}
\mathbb{T}_{i_{1} i_{1}} d_{i_{1}} & \mathbb{T}_{i_{2} i_{1}} d_{i_{2}} & \ldots & \mathbb{T}_{i_{n} i_{1}} d_{i_{n}} \\
-\mathbb{T}_{i_{1} i_{2}} \widetilde{d}_{i_{1}} & \mathbb{T}_{i_{2} i_{2}} d_{i_{2}} & \ldots & \mathbb{T}_{i_{n} i_{2}} d_{i_{n}} \\
\vdots & \vdots & \vdots & \vdots \\
-\mathbb{T}_{i_{1} i_{n}} \widetilde{d}_{i_{1}} & -\mathbb{T}_{i_{2} i_{n}} \widetilde{d}_{i_{2}} & \ldots & \mathbb{T}_{i_{n} i_{n}} d_{i_{n}}
\end{array}\right|
$$

Here and to end of this appendix the $\omega$ dependence is omitted for conciseness. The factors $d_{i}$ and $\widetilde{d}_{i}$ are given by (32) and (A6), and the matrix $\mathbb{T}$, generated by (B1), is defined in terms of $\mathbb{S}$ by

$$
\begin{aligned}
& \mathbb{T}_{11}=-\mathbb{T}_{22}=\left|\mathbb{S}_{12}\right|^{2} \equiv \tau, \\
& \mathbb{T}_{12}=\overline{\mathbb{T}}_{21}=-\mathbb{S}_{11} \overline{\mathbb{S}}_{21} .
\end{aligned}
$$

In order to compute $\mathbb{D}_{n}$ we introduce an auxiliary algebra of fermionic oscillators generated by $\left\{a_{i}, a_{i}^{*}: i=1,2\right\}$, which satisfy

$$
\left[a_{i}, a_{j}^{*}\right]_{+}=\delta_{i j}, \quad\left[a_{i}, a_{j}\right]_{+}=\left[a_{i}^{*}, a_{j}^{*}\right]_{+}=0 .
$$

Let us consider the quadratic operators

$$
L=\sum_{i=1}^{2} \gamma_{i} a_{i}^{*} a_{i}, \quad J=\sum_{i, j=1}^{2} a_{i}^{*} \mathbb{T}_{i j} a_{j}
$$

The key observation now is that $\mathbb{D}_{n}$ can be represented in the form

$$
\mathbb{D}_{n}=\frac{\operatorname{Tr}\left(\mathrm{e}^{-L} J^{n}\right)}{\operatorname{Tr}\left(\mathrm{e}^{-L}\right)},
$$

which can be verified by explicit computation using (B5) and (B6). One has at this point that

$$
\sum_{n=0}^{\infty} \frac{(\mathrm{i} \eta)^{n}}{n !} \mathbb{D}_{n}=\frac{\operatorname{Tr}\left(\mathrm{e}^{-L} \mathrm{e}^{\mathrm{i} \eta J}\right)}{\operatorname{Tr}\left(\mathrm{e}^{-L}\right)} .
$$

The right-hand side of (B8) has been previously computed [50] for the full counting statistics of the particle current (25). Using the result of Ref. [50], one finds

$$
\sum_{n=0}^{\infty} \frac{(\mathrm{i} \eta)^{n}}{n !} \mathbb{D}_{n}=1+\mathrm{i} c_{1} \sqrt{\tau} \sin (\eta \sqrt{\tau})+c_{2}[\cos (\eta \sqrt{\tau})-1]
$$

where $c_{i}$ are defined by (45). From (B9) it follows that

$$
\mathbb{D}_{n}=\left\{\begin{array}{lll}
1, & n=0, & \\
\tau^{k} c_{1} & n=2 k-1, & k=1,2, \ldots, \\
\tau^{k} c_{2}, & n=2 k, & k=1,2, \ldots
\end{array}\right.
$$

implying the result (43) and (44).
[1] H. Spohn and J. L. Lebowitz, in Advances in Chemical Physics: For Ilya Prigogine, edited by S. A. Rice (John Wiley and Sons, New York, 1978), Vol. 38, p. 109.

[2] I. Ojima, H. Hasegawa, and M. Ichijanagi, J. Stat. Phys. 50, 633 (1988).

[3] V. Jakšić and C.-A. Pillet, Comm. Math. Phys. 217, 285 (2001).

[4] C. Maes and K. Netoĉcný, J. Stat. Phys. 110, 269 (2003).

[5] W. H. Aschbacher and H. Spohn, Lett. Math. Phys. 75, 17 (2006).

[6] R. C. Dewar, C. H. Lineweaver, R. K. Niven, and K. RegenauerLieb, Beyond the Second Law (Springer, Heidelberg, 2014).

[7] G. Nenciu, J. Math. Phys. 48, 033302 (2007).

[8] S. Deffner and E. Lutz, Phys. Rev. Lett. 107, 140404 (2011).

[9] P. Gaspard, New J. Phys. 17, 045001 (2015).

[10] K. Funo, T. Shitara, and M. Ueda, Phys. Rev. E 94, 062112 (2016).
[11] J.-P. Eckmann, C.-A. Pillet, and L. Rey-Bellet, J. Stat. Phys. 95, 305 (1999).

[12] C. Maes, F. Redig, and A. Van Moffaert, J. Math. Phys. 41, 1528 (2000).

[13] J. M. R. Parrondo, C. van den Broeck, and R. Kawai, New J. Phys. 11, 073008 (2009).

[14] H. J. D. Miller and J. Anders, Phys. Rev. E 95, 062123 (2017).

[15] S. Borlenghi, S. Iubini, S. Lepri, and J. Fransson, Phys. Rev. E 96, 012150 (2017).

[16] A. Dechant and S. Sasa, arXiv:1708.08653.

[17] G. E. Crooks, Phys. Rev. E 60, 2721 (1999).

[18] C. Jarzynski, J. Statist. Phys. 98, 77 (2000).

[19] D. J. Evans and D. J. Searles, Adv. Phys. 51, 1529 (2002).

[20] U. Seifert, Phys. Rev. Lett. 95, 040602 (2005).

[21] K. Saito and Y. Utsumi, Phys. Rev. B 78, 115429 (2008). 
[22] N. Merhav and Y. Kafri, J. Stat. Mech: Theory Exp. (2010) P12022.

[23] C. Jarzynski, Ann. Rev. Cond. M. Phys. 2, 329 (2011).

[24] C. L. Kane and M. P. A. Fisher, Phys. Rev. B 46, 15233 (1992).

[25] C. Nayak, M. P. A. Fisher, A. W. W. Ludwig, and H. H. Lin, Phys. Rev. B 59, 15694 (1999).

[26] S. Lal, S. Rao, and D. Sen, Phys. Rev. B 66, 165327 (2002).

[27] S. Chen, B. Trauzettel, and R. Egger, Phys. Rev. Lett. 89, 226404 (2002).

[28] C. Chamon, M. Oshikawa, and I. Affleck, Phys. Rev. Lett. 91, 206403 (2003).

[29] B. Bellazzini, P. Calabrese, and M. Mintchev, Phys. Rev. B 79, 085122 (2009).

[30] P. A. Camati, J. P. S. Peterson, T. B. Batalhão, K. Micadei, A. M. Souza, R. S. Sarthour, I. S. Oliveira, and R. M. Serra, Phys. Rev. Lett. 117, 240502 (2016).

[31] M. Brunelli et al., arXiv:1602.06958.

[32] R. Landauer, IBM J. Res. Dev. 1, 223 (1957); Philos. Mag. 21, 863 (1970).

[33] M. Büttiker, Phys. Rev. Lett. 57, 1761 (1986); IBM J. Res. Dev. 32, 317 (1988).

[34] P. W. Anderson, D. J. Thouless, E. Abrahams, and D. S. Fisher, Phys. Rev. B 22, 3519 (1980).

[35] D. S. Fisher and P. A. Lee, Phys. Rev. B 23, 6851 (1981).

[36] U. Sivan and Y. Imry, Phys. Rev. B 33, 551 (1986).

[37] Th. Martin and R. Landauer, Phys. Rev. B 45, 1742 (1992).

[38] M. Büttiker, Phys. Rev. B 46, 12485 (1992).

[39] M. J. M. de Jong and C. W. J. Beenakker, Shot noise in mesoscopic systems, in Mesoscopic Electron Transport, edited by L. L. Sohn, L. P. Kouwenhoven, and G. Schoen, NATO ASI Series 345 (Kluwer Academic Publishers, Dordrecht, 1997), p. 225.

[40] Ya. M. Blanter and M. Büttiker, Phys. Rep. 336, 1 (2000).

[41] M. Mintchev, L. Santoni, and P. Sorba, J. Phys. A 48, 285002 (2015).

[42] M. Mintchev, L. Santoni, and P. Sorba, Ann. Phys. 529, 1600274 (2017).

[43] V. K. Khlus, Sov. Phys. JETP 66, 1243 (1987) [Zh. Eksp. Teor. Fiz. 93, 2179 (1987)].

[44] G. B. Lesovik, JETP Lett. 49, 592 (1989) [Pis'ma. Zh. Eksp. Teor. Fiz. 49, 513 (1989)].
[45] L. S. Levitov and G. B. Lesovik, JETP Lett. 55, 555 (1992) [Pis'ma. Zh. Eksp. Teor. Fiz. 55, 534 (1992)].

[46] L. S. Levitov, H. Lee, and G. B. Lesovik, J. Math. Phys. 37, 4845 (1996).

[47] G. B. Lesovik and N. M. Chtchelkatchev, JETP Lett. 77, 393 (2003) [Pis'ma. Zh. Eksp. Teor. Fiz. 77, 464 (2003)].

[48] D. B. Gutman, Y. Gefen, and A. D. Mirlin, High cumulants of current fluctuations out of equilibrium, in Quantum Noise in Mesoscopic Physics, edited by Y. V. Nazarov (Kluwer Academic Publishers, Dordrecht, 2003), p. 497.

[49] M. Esposito, U. Harbola, and S. Mukamel, Rev. Mod. Phys. 81, 1665 (2009).

[50] M. Mintchev, L. Santoni, and P. Sorba, J. Phys. A 49, 265002 (2016).

[51] M. Mintchev, L. Santoni, and P. Sorba (unpublished).

[52] H. B. Callen, Thermodynamics and an Introduction to Thermostatistics (John Wiley and Sons, Hoboken, NJ, 1960).

[53] M. Mintchev, L. Santoni, and P. Sorba, J. Phys. A 48, 055003 (2015).

[54] R. Landauer, Nature (London) 392, 658 (1998).

[55] A. Kumar, L. Saminadayar, D. C. Glattli, Y. Jin, and B. Etienne, Phys. Rev. Lett. 76, 2778 (1996).

[56] D. E. Feldman and M. Heiblum, Phys. Rev. B 95, 115308 (2017).

[57] V. Kostrykin and R. Schrader, Fortschr. Phys. 48, 703 (2000).

[58] M. Harmer, J. Phys. A 33, 9015 (2000).

[59] P. Kuchment, Proc. Symp. Pure. Math, 104, 13 (2012).

[60] D. Bernard, B. Doyon, and J. Viti, J. Phys. A 48, 05FT01 (2015).

[61] P. Calabrese, M. Mintchev, and E. Vicari, J. Phys. A 45, 105206 (2011).

[62] M. Mintchev, J. Phys. A 44, 415201 (2011).

[63] E. Wigner, Phys. Rev. 40, 749 (1932).

[64] K. E. Cahill and R. J. Glauber, Phys. Rev. 177, 1882 (1969).

[65] H.-Y. Fan, Phys. Lett. A 294, 134 (2002).

[66] P. P. Hofer and A. A. Clerk, Phys. Rev. Lett. 116, 013603 (2016).

[67] P. P. Hoffer, Quantum 1, 32 (2017).

[68] J. A. Shohat and J. D. Tamarkin, The Problem of Moments (American Mathematical Society, Providence, RI, 1970).

[69] M. Mintchev and P. Sorba, J. Phys. A 46, 095006 (2013).

[70] K. Gawedki and C. Tauber, Nucl. Phys. B 896, 138 (2015). 